Elevated cardiovascular risk factors in Multiple Sclerosis

Peer-reviewed author version

KEYTSMAN, Charly; OP 'T EIJNDE, Bert; HANSEN, Dominique; VERBOVEN, Kenneth \& WENS, Inez (2017) Elevated cardiovascular risk factors in Multiple Sclerosis. In: Multiple Sclerosis and Related Disorders, 17, p. 220-223.

DOI: 10.1016/j.msard.2017.08.011

Handle: http://hdl.handle.net/1942/25020 


\section{Elevated cardiovascular risk factors in Multiple Sclerosis.}

Charly Keytsman, Bert O Eijnde, Dominique Hansen, Kenneth Verboven, Inez

\section{Wens}

REVAL Rehabilitation Research Center, BIOMED Biomedical Research Institute, Faculty of Medicine and Life Sciences, Hasselt University, Agoralaan Building A, Diepenbeek, Belgium

Corresponding author:

Charly Keytsman

REVAL Rehabilitation Center - Biomedical Research Institute (BIOMED) - Hasselt University

Agoralaan Building A, B-3590 Diepenbeek, Belgium

Email: charly.keytsman@uhasselt.be

Tel: $+32477 / 611122$ 


\section{ABSTRACT}

Background. Multiple sclerosis (MS) is associated with elevated cardiovascular mortality. To prevent this a better understanding of their CVD risk factors and interrelations is necessary.

Methods. MS patients $(n=52)$ and healthy controls $(H C, n=24)$ were matched for age, height, weight, body mass index and physical activity. Body composition, resting blood pressure (BP), resting heart rate (HR), glucose tolerance, HbA1c, blood lipids (HDL, LDL, total cholesterol, triglyceride concentrations) and c-reactive protein concentrations were analyzed. Regression analyses identified independent CVD risk factors and their interrelations in MS

Results. In MS and compared to $\mathrm{HC}$, fat mass $(25.1 \pm 1.2 \mathrm{~kg}$ vs. $17.9 \pm 1 \mathrm{~kg})$, fat percentage $(33.8 \pm 1.2 \%$ vs. $28.4 \pm 1.5 \%)$, systolic $(130 \pm 1.8 \mathrm{mmHg}$ vs. $120 \pm 2.9 \mathrm{mmHg})$ and diastolic $(79 \pm 1.1 \mathrm{mmHg}$ vs. $71 \pm 1.9 \mathrm{mmHg}) \mathrm{BP}$, resting HR $(72 \pm 1.4 \mathrm{bpm}$ vs. $60 \pm 2 \mathrm{bpm})$, blood triglycerides $(113.8 \pm 8.6 \mathrm{mg} / \mathrm{dl}$ vs. $98.2 \pm 17.4 \mathrm{mg} / \mathrm{dl})$, fasting $(13.5 \pm 2.9 \mathrm{mU} / \mathrm{l}$ vs. $7.2 \pm 0.8 \mathrm{mU} / \mathrm{I})$ and $2 \mathrm{~h}$ insulin $(71.9 \pm 12.5 \mathrm{mU} / \mathrm{I}$ vs. $35.8 \pm 8.1 \mathrm{mU} / \mathrm{I}), 2 \mathrm{~h}$ glucose $(6.3 \pm 0.5 \mathrm{mmol} / \mathrm{I}$ vs. $4.8 \pm 0.5 \mathrm{mmol} / \mathrm{I})$ and HOMA index (3.7 \pm 1.1 vs. $1.7 \pm 0.2)$ were significantly $(p<0.05)$ elevated. Total cholesterol, blood HDL and LDL concentrations did nog differ between groups $(p<0.05)$. Regression analyses indicated that MS is independently associated with elevated fat mass/percentage, systolic and diastolic BP and HR and in MS fat mass appears to be an independent contributor of the other measured CVD risk factors in MS.

Conclusion. Persons with MS have an increased risk for CVD and fat mass appears to be an important risk factor. Therefore, normalizing whole body fat should be an essential part of MS treatment.

Keywords: multiple sclerosis, cardiovascular diseases, body composition, fat mass, mortality 


\section{INTRODUCTION}

MS patients display a reduced exercise capacity (Bosnak-Guclu et al., 2012; Langeskov-Christensen et al., 2015), impaired muscle strength (Carroll et al., 2005; Wens et al., 2014) and overall functional capacity (Bosnak-Guclu et al., 2012; Ellis and Motl, 2013; Kjolhede et al., 2015; Savci et al., 2005). This results in a sedentary lifestyle and thus further deconditioning. The latter leads to progressive worsening of fatigue and muscle weakness, reduced exercise and functional capacity and deterioration of various neurological symptoms, independently of the disease process per se (Ellis and Motl, 2013; Motl et al., 2008). In healthy persons, such a sedentary lifestyle is associated with an increased overall risk to develop cardiovascular diseases (CVD) (Buchan et al., 2012; Warren et al., 2010). In MS the likelihood for cardiovascular mortality is significantly greater compared to the general population (HR: 1.29, (Manouchehrinia et al., 2016). To improve survival in persons with MS, it is thus relevant to explore what CVD factors are primarily affected in this population.

We have previously shown that CVD risk factors may be elevated in MS compared to healthy controls (HC) (Wens et al., 2013b). However, in MS it is still unclear whether an increased CVD risk is primarily mediated by changes in body composition, blood pressure, blood lipid profile or glycemic control (Wens et al., 2013b). More recently Moccia et al. (Moccia et al., 2015) investigated the CVD risk in a large cohort of persons with MS and reported that the CVD risk was comparable to healthy controls. This study applied the Framingham Risk Score (FRS) to assess CVD risk, in which sex, age, smoking behavior, blood pressure, blood glucose concentrations and body mass index (BMI) are taken into account (D'Agostino et al., 2008; Mahmood et al., 2014). However, it is well known that in persons with MS the use of BMI is not recommended because it underestimates adiposity in this population (Pilutti and Motl, 2016). Furthermore, the FRS does not take into account various other important CVD risk factors such as body composition and blood lipid profile. Finally, FRS is not validated in MS yet. Thus, whether and how, CVD risk is elevated in MS remains unclear but is of great clinical importance.

Therefore the present study investigates all common CVD risk factors in MS simultaneously and explores their interrelations. 


\section{MATERIALS \& METHODS}

\subsection{Subjects}

Patients with MS ( $n=52$, mean EDSS $=2.9 \pm 0.2$, range 0 to 5.5$)$ and healthy controls $(H C, n=24)$ were matched for age, height, weight, body mass index (BMI), physical activity, and were included following written informed consent and aged $>18$ years. Subjects were excluded if they participated in another study, had (in case of MS) an acute exacerbation 6 months prior to the start of the study or had an EDSS score $>6$. Subjects were recruited following local advertisement. Phenotypes of MS (RR; relapsing remitting, PP; primary progressive, SP; secondary progressive) and the use of disease modifying therapies (DMT) were inventoried. The study was approved by the local Ethical Committee of the Jessa hospital and Hasselt University, and was performed in accordance with the Declaration of Helsinki. This study was registered at ClinicalTrials.gov (NCT02466165).

\subsection{Study Design}

In this cross-sectional study, body composition, resting blood pressure and heart rate, and blood HDL, LDL, total cholesterol, triglyceride concentrations, c-reactive protein, glucose- and insulin concentrations were assessed. Furthermore, all subjects were asked whether they smoked and used medication to regulate blood pressure, cholesterol or glucose tolerance.

\subsection{Measurements}

\subsubsection{Body composition:}

Whole body fat and lean tissue mass (with exclusion of the head) were assessed using Dual Energy X-ray Absorptiometry scan (DEXA, Hologic Series Delphi-A Fan Beam X-ray Bone Densitometer, Vilvoorde, Belgium) and a calibrated analogue weight scale $\left(\mathrm{Seca}^{\circledR}\right)$ was used to measure total body mass. Android fat mass and the ratio of android fat mass to total fat mass were evaluated. 


\subsubsection{Blood pressure and heart rate:}

Resting blood pressure and heart rate were measured with an automatic blood pressure cuff (Omron M4-I, Omron Healthcare Europe B.V., Hoofddorp, The Netherlands) in a supine position (7min rest) immediately following the DEXA scan (to prevent orthostatic effects).

\subsubsection{Oral glucose tolerance test:}

Glycemic control was investigated using an oral glucose tolerance test (OGTT). Following a 10h overnight fast, all participants received a $1 \mathrm{~g}$ glucose/kg body weight solution (300ml, (Wens et al., 2013a). Before and after glucose administration, capillary blood samples were collected from a hyperaemic earlobe at $30 \mathrm{~min}$ intervals (2h), to measure blood glucose concentrations immediately (Analox, GM7 Micro-stat, Analox instruments Ltd, London, UK). Blood glucose concentrations were converted to plasma concentrations using a multiplier of 1.11 (D'Orazio et al., 2006). To determine serum insulin concentrations, $4 c c$ of venous blood was collected in serum separation tubes (SST, BD Vacutainer ${ }^{\circ}$, Becton Dickinson, Erembodegem, Belgium) at $1 \mathrm{~h}$ intervals. After 30min, allowing blood coagulation, samples were centrifuged during $10 \mathrm{~min}$ at $3500 \mathrm{rpm}$. The obtained serum was frozen and stored at $-80^{\circ} \mathrm{C}$ for batch analysis of serum insulin concentrations (Mercodia Insulin ELISA ${ }^{\circ}$, Uppsala, Sweden).

\subsubsection{Cardiovascular risk factors: blood analysis}

Following an overnight fasting period (10h), a venous blood sample was collected and centrifuged at $2000 \mathrm{rpm}$ for $10 \mathrm{~min}$. Plasma was frozen immediately in liquid nitrogen and stored at $-80^{\circ} \mathrm{C}$ until further analysis. Plasma samples were analyzed for blood insulin, total cholesterol, high-density lipoprotein cholesterol (HDL), lowdensity lipoprotein cholesterol (LDL), triglyceride concentrations, C-reactive protein (CRP, Beckman Synchron LX 20 Analyzer $^{\circledast}$, Beckman Coulter Inc., Diamond Diagnostics, USA), and glycosylated haemoglobin (HbA1c) (Hi-Auto A1c Analyzer ${ }^{\varpi}$, Menarini Diagnostics Inc., Florence, Italy) concentrations. The Homeostasis Model Assessment (HOMA-IR: fasting blood glucose [mmmol/I] x fasting serum insulin [mU/I]/22.5, (Sarafidis et al., 2007) was used to estimate/calculate insulin resistance (IR) and $\beta$-cell function from fasting glucose and insulin concentrations. This assessment model indicates a normal insulin sensitivity with a score between 1.7 and 2, whereas a score of $>2$ suggests altered insulin sensitivity. 


\subsubsection{Physical activity}

Participants were asked to report their physical activity level (Physical Activity Scale for Individuals with Physical Disabilities, PASIPD,(Washburn et al., 2002). The PASIPD is a 13-item physical activity questionnaire involving participation in household, recreational and occupational activities over the past 7 days. The scores are the average hours of participation daily multiplied with a metabolic equivalent value.

\subsection{Statistical Analysis}

All data were analyzed using SPPS v. 22.0 (IBM) and checked for normality (Shapiro-Wilk test). Data was not normally distributed and differences between groups were analyzed using an unpaired student's t-test (Mann Whitney U test). Fat mass, lean body mass, total mass, fat percentage, systolic and diastolic blood pressure, resting heart rate, blood total cholesterol, $\mathrm{HDL}, \mathrm{LDL}$ and triglyceride concentrations, $\mathrm{CRP}$, fasting and $2 \mathrm{~h}$ blood glucose concentrations, $\mathrm{HbA} 1 \mathrm{c}$, fasting and $2 \mathrm{~h}$ blood insulin concentrations and HOMA index were inserted into a multivariate regression model to examine relations between CVD risk factors and the presence of MS, taking age, gender, physical activity, smoking behavior and medication for blood pressure, cholesterol and glucose tolerance into account. Next, multivariate regression analyses were applied to examine interrelations between CVD risk factors, in which for every deviant CVD risk factor a model was created that examined relations with all other CVD risk factors, in MS only. All data are presented as means \pm SEM and considered statistically significant when $\mathrm{p}<0.05$ (2-tailed).

\section{RESULTS}

\subsection{Subject characteristics}

Gender differences and differences in smoking behavior were present between groups $(p<0.05$, Table 1$)$. Medication intake for blood pressure, cholesterol and glucose tolerance were higher in MS. Age, height, weight, BMI and physical activity did not differ between groups. Relapsing-remitting MS was diagnosed in 37 patients, whilst 2 patients suffered from primary-progressive MS and 13 patients from the secondary-progressive form. 
Forty-five MS patients were on disease modifying therapy, of which 7 on interferon-beta, 6 on glatiramer acetate, 14 on natalizumab, 4 on dimethyl fumarate, 5 on teriflunomide and 9 on fingolimod.

Table 1. Subject characteristics

\begin{tabular}{|c|c|c|}
\hline & $\mathrm{HC}(n=24)$ & $M S(n=52)$ \\
\hline Age (y) & $48.6 \pm 2.5$ & $51.2 \pm 1.5$ \\
\hline Height (m) & $1.7 \pm 0.0$ & $1.7 \pm 0.0$ \\
\hline Weight (kg) & $69.9 \pm 2.9$ & $72.6 \pm 1.9$ \\
\hline BMI & $24.2 \pm 0.5$ & $25.7 \pm 0.6$ \\
\hline PASIPD & $21.7 \pm 2.2$ & $17.9 \pm 1.8$ \\
\hline Gender $(f / m)$ & $15 / 9$ & $36 / 16^{*}$ \\
\hline EDSS & - & $2.9 \pm 0.2$ \\
\hline Med. BP $(n=)$ & 2 & $14^{*}$ \\
\hline Med. Cholesterol $(n=)$ & 2 & $10^{*}$ \\
\hline Med. glucose tolerance $(n=)$ & 0 & $2^{*}$ \\
\hline Smoking $(n=)$ & 2 & $7 *$ \\
\hline Interferon-beta & - & 7 \\
\hline Glatiramer acetate & - & 6 \\
\hline Natalizumab & - & 14 \\
\hline Dimethylfumaraat & - & 4 \\
\hline Teriflunomide & - & 5 \\
\hline Fingolimod & - & 9 \\
\hline RRMS & - & 37 \\
\hline PPMS & - & 2 \\
\hline SPMS & - & 13 \\
\hline
\end{tabular}

Data are expressed as means \pm SEM and represent subject characteristics of healthy controls and MS patients. Abbreviations: $f$, female; $m$, male; $B M I$, Body Mass Index; EDSS, Expanded Disability Status Scale; PASIPD, Physical Activity Scale for Individuals with Physical Disabilities. Med, medication. DMT; Disease Modifying Therapy. RRMS; Relapsing Remitting MS. PPMS; Primary Progressive MS. SPMS; Secondary Progressive MS. ${ }^{*} p<0.05$ compared to $H C$. 
In MS patients, body composition variables such as fat mass $(+40 \%)$ and body fat percentage $(+19 \%)$ were significantly elevated compared to HC (Table 2$)$ with significantly greater $(p<0.05)$ android $(21.8 \pm 1.2 \mathrm{~kg}$ vs. $14,7 \pm 1.2 \mathrm{~kg})$ fat mass and a significantly $(p<0.05)$ higher ratio of android to total fat mass $(0.96 \pm 0.0$ vs. $0.80 \pm 0.0)$ in $\mathrm{MS}$ (data not shown). Furthermore, systolic (+8\%) and diastolic blood pressure (+11\%), resting heart rate $(+20 \%)$, blood triglyceride concentrations $(+16 \%), 2 \mathrm{~h}$ glucose $(+31 \%)$, fasting $(+87 \%)$ and $2 \mathrm{~h}$ insulin $(+101 \%$ ) concentrations and HOMA index (+117\%) were significantly elevated in MS patients compared to HC. Blood total cholesterol, blood HDL and LDL concentrations were not different ( $p>0.05)$ between groups. 
Table 2. Cardiovascular risk factors in multiple sclerosis and healthy controls

\begin{tabular}{|c|c|c|c|}
\hline & HC & MS & p-value \\
\hline Fat mass (kg) & $17.9 \pm 1$ & $22.6 \pm 1.2^{*}$ & 0.01 \\
\hline Lean body mass (kg) & $45.9 \pm 2.4$ & $43.6 \pm 1.4$ & 0.37 \\
\hline Total mass (kg) & $63.7 \pm 2.6$ & $66.3 \pm 1.9$ & 0.42 \\
\hline Fat percentage (\%) & $28.4 \pm 1.5$ & $33.8 \pm 1.2^{*}$ & 0.01 \\
\hline Systolic BP (mmHg) & $120 \pm 2.9$ & $130 \pm 1.8^{*}$ & 0.001 \\
\hline Diastolic PB (mmHg) & $71 \pm 1.9$ & $79 \pm 1.1^{*}$ & 0.001 \\
\hline$H_{\text {rest }}(\mathrm{bpm})$ & $60 \pm 2$ & $72 \pm 1.4^{*}$ & 0.000 \\
\hline Cholesterol $_{\text {Total }}(\mathrm{mg} / \mathrm{dl})$ & $190.1 \pm 7.6$ & $199.7 \pm 4.8$ & 0.18 \\
\hline LDL (mg/dl) & $110.5 \pm 6.2$ & $112.1 \pm 4.2$ & 0.95 \\
\hline HDL (mg/dl) & $62.3 \pm 3.6$ & $65.6 \pm 2.6$ & 0.69 \\
\hline Triglycerides (mg/dl) & $98.2 \pm 17.4$ & $113.8 \pm 8.6^{*}$ & 0.02 \\
\hline CRP (mg/dl) & $2.4 \pm 0.7$ & $5.4 \pm 1.8$ & 0.27 \\
\hline Fasting glucose (mmol/l) & $5.3 \pm 0.2$ & $5.5 \pm 0.1$ & 0.80 \\
\hline $2 \mathrm{~h}$ glucose $(\mathrm{mmol} / \mathrm{I})$ & $4.8 \pm 0.5$ & $6.3 \pm 0.5^{*}$ & 0.01 \\
\hline HbA1c (\%) & $5.2 \pm 0.1$ & $5.3 \pm 0.1$ & 0.61 \\
\hline Fasting insulin (mU/I) & $7.2 \pm 0.8$ & $13.5 \pm 2.9^{*}$ & 0.000 \\
\hline $2 \mathrm{~h}$ insulin (mU/l) & $35.8 \pm 8.1$ & $71.9 \pm 12.5^{*}$ & 0.03 \\
\hline HOMA index & $1.7 \pm 0.2$ & $3.7 \pm 1.1^{*}$ & 0.001 \\
\hline
\end{tabular}

Data are expressed as means \pm SEM and represent various cardiovascular risk factors of healthy controls (HC) and MS patients (MS). Body composition was determined with exclusion of the head. Abbreviations: $B P$, blood pressure; $H R$, heart rate; bpm, beats per minute. ${ }^{*} p<0.05$ compared to $H C$.

\subsection{Regression analysis}

When adjusted for age, gender, physical activity, smoking behavior and medication for blood pressure, cholesterol and glucose tolerance, MS was independently related $(p<0.05)$ to elevated fat percentage and fat mass, systolic and diastolic blood pressure and resting heart rate. 
Furthermore, after investigating the interrelations between CVD risk factors, in which for every deviant CVD risk factor a model was created that examined relations with all other CVD risk factors, fat mass appeared to be independently related $(p<0.05)$ to fat percentage, total mass, lean tissue mass, diastolic blood pressure, blood triglyceride and CRP concentrations, resting heart rate, blood $\mathrm{HbA} 1 \mathrm{c}, 2 \mathrm{~h}$ blood glucose and HDL concentrations and a trend for systolic blood pressure $(P=0.05)$.

\section{DISCUSSION}

This study shows that MS is independently associated with several abnormal CVD risk factors. This probably increases their risk to develop CVD. Interestingly, following regression analyses fat mass appears to be an important contributing factor for this.

To our knowledge the present study is the first to investigate all common CVD risk factors in MS (Wens et al., 2013b) simultaneously. Following inter-group comparisons we now show that MS patients exhibit greater whole body fat mass and fat percentage, higher blood pressure (systolic and diastolic), resting heart rates, blood triglyceride concentrations, a disturbed whole body glucose tolerance and disturbed insulin concentrations compared to HC. Consequently, MS patients are probably more prone to develop future (preventable) cardiovascular complications and/or comorbidities. The present findings contrast with a recently performed study of Moccia et al. (Moccia et al., 2015) investigating the risk to develop CVD in MS using the FRS (D'Agostino et al., 2008; Mahmood et al., 2014). This risk score comprises an algorithm that estimates the 10-year likelihood to develop CVD including risk factors such as age, gender, smoking status, body mass index, systolic blood pressure, glucose tolerance and use of antihypertensive medication. Moccia et al. (Moccia et al., 2015) concluded that, based on the FRS, CVD risk was normal in MS patients. The discrepancy with our findings probably has several reasons. First, we have included a wider range of CVD risk factors such as fat mass, fat free mass and blood cholesterol. These risk factors have been shown to correlate better with CVD incidence than the Framingham Score factors (Buchan et al., 2012) and therefore they are probably more predictive. Second, an important component of the FRS algorithm is the BMI (D'Agostino et al., 2008; Mahmood et al., 2014). However in MS, Pilutti (Pilutti and Motl, 2016) et al. clearly showed that BMI significantly underestimates the amount of adipose tissue. Consequently, we have matched MS patients and HC for BMI and clearly demonstrate significantly 
higher total body fat mass and fat percentage compared to HC. This shows that screening MS patients using BMI or predictive calculations that include BMI probably underestimates their actual risk to develop CVD. Under the conditions of the present study in MS blood lipid profile (total cholesterol, HDL, LDL) was not altered compared to $\mathrm{HC}$ and may thus be an inferior CVD predictor in this population.

It is important to note that age, smoking, gender, medication for blood pressure, cholesterol and glucose tolerance (Mosca et al., 2011) may importantly contribute to the above described findings. In the current study more persons with MS smoked, used medication for blood pressure, cholesterol and glucose tolerance and gender differences were present between groups. To exclude the effect of these confounding factors and investigate the sole impact of MS on CVD risk we performed regression analyses and interestingly demonstrate that irrespective of age, gender, physical activity, smoking behavior, medication for blood pressure, cholesterol and glucose tolerance, a higher fat percentage, fat mass, systolic and diastolic blood pressure as well as resting heart rate are independently related to MS. In a second series of regression analyses, we then examined the interrelations between these CVD risk factors, in which for every deviant CVD risk factor (fat mass, fat percentage, diastolic and systolic blood pressure and resting heart rate ) a model was created that examined relations with all other CVD risk factors. We now demonstrate that in MS, fat mass is independently related to systolic and diastolic blood pressure, resting heart rate but also to blood HDL, CRP, $2 \mathrm{~h}$ glucose, HbA1c, CRP and triglyceride concentrations. This suggests that fat mass is a very important CVD risk factor in MS. Furthermore, it is important to notice that persons with MS in this study presented significantly more android fat mass, as well as higher ratios of android to total fat mass ratios. This indicates the presence of more visceral adipose tissue in MS, which is known to be associated with increased cardiovascular risk factors (Matsushita et al., 2014). In recent years, the role of adipose tissue in the pathogenesis of MS has become subject of great interest (Guerrero-Garcia et al., 2016). It is known that elevated fat tissue causes an increase in the production of adipokines, which are involved in the regulation of various physiological processes, such as energy balance and insulin sensitization, and the immune response. As such, it is believed that increased fat mass, and thus elevated levels of adipokines, may be involved in the altered immune response and inflammatory processes in MS. Therefore, increased fat mass may not only affect cardiovascular risk in MS but may also negatively influence MS progress and treatment response as reported elsewhere (Guerrero-Garcia et al., 2016). 
Our findings may thus indicate that multidisciplinary programs directed to prevent/treat (cardiovascular) complications and mortality in MS should also include strategies that lower lowering fat mass.

Limitations

Although not significant, it is important to note that a 1.5-unit difference in BMI between MS and HC was observed in this study. This may cause the potential for residual confounding.

\section{CONCLUSION}

Persons with MS are probably more prone to an increased risk for CVD and fat mass appears to be a very important mediating risk factor. Therefore, MS treatment should also include interventions that restore/optimize whole body fat mass.

\section{Acknowledgements}

We thank the MS Liga Flanders for partly funding this project.

\section{Conflicts of interest}

The authors declare that they have no conflict of interest. 
Bosnak-Guclu, M., Gunduz, A.G., Nazliel, B., Irkec, C., 2012. Comparison of functional exercise capacity, pulmonary function and respiratory muscle strength in patients with multiple sclerosis with different disability levels and healthy controls. J Rehabil Med 44(1), 80-86.

Buchan, D.S., Thomas, N.E., Baker, J.S., 2012. Novel risk factors of cardiovascular disease and their associations between obesity, physical activity and physical fitness. J Public Health Res 1(1), 59-66. Carroll, C.C., Gallagher, P.M., Seidle, M.E., Trappe, S.W., 2005. Skeletal muscle characteristics of people with multiple sclerosis. Arch Phys Med Rehabil 86(2), 224-229.

D'Agostino, R.B., Sr., Vasan, R.S., Pencina, M.J., Wolf, P.A., Cobain, M., Massaro, J.M., Kannel, W.B., 2008. General cardiovascular risk profile for use in primary care: the Framingham Heart Study. Circulation 117(6), 743-753.

D'Orazio, P., Burnett, R.W., Fogh-Andersen, N., Jacobs, E., Kuwa, K., Kulpmann, W.R., Larsson, L., Lewenstam, A., Maas, A.H., Mager, G., Naskalski, J.W., Okorodudu, A.O., 2006. Approved IFCC recommendation on reporting results for blood glucose: International Federation of Clinical Chemistry and Laboratory Medicine Scientific Division, Working Group on Selective Electrodes and Point-of-Care Testing (IFCC-SD-WG-SEPOCT). Clin Chem Lab Med 44(12), 1486-1490.

Ellis, T., Motl, R.W., 2013. Physical activity behavior change in persons with neurologic disorders: overview and examples from Parkinson disease and multiple sclerosis. J Neurol Phys Ther 37(2), 8590.

Guerrero-Garcia, J.J., Carrera-Quintanar, L., Lopez-Roa, R.I., Marquez-Aguirre, A.L., Rojas-Mayorquin, A.E., Ortuno-Sahagun, D., 2016. Multiple Sclerosis and Obesity: Possible Roles of Adipokines. Mediators Inflamm 2016, 4036232.

Kjolhede, T., Vissing, K., Langeskov-Christensen, D., Stenager, E., Petersen, T., Dalgas, U., 2015. Relationship between muscle strength parameters and functional capacity in persons with mild to moderate degree multiple sclerosis. Mult Scler Relat Disord 4(2), 151-158.

Langeskov-Christensen, M., Heine, M., Kwakkel, G., Dalgas, U., 2015. Aerobic capacity in persons with multiple sclerosis: a systematic review and meta-analysis. Sports Med 45(6), 905-923.

Mahmood, S.S., Levy, D., Vasan, R.S., Wang, T.J., 2014. The Framingham Heart Study and the epidemiology of cardiovascular disease: a historical perspective. Lancet 383(9921), 999-1008. Manouchehrinia, A., Tanasescu, R., Tench, C.R., Constantinescu, C.S., 2016. Mortality in multiple sclerosis: meta-analysis of standardised mortality ratios. J Neurol Neurosurg Psychiatry 87(3), 324331.

Matsushita, Y., Nakagawa, T., Yamamoto, S., Kato, T., Ouchi, T., Kikuchi, N., Takahashi, Y., Yokoyama, T., Mizoue, T., Noda, M., 2014. Adiponectin and visceral fat associate with cardiovascular risk factors. Obesity (Silver Spring, Md.) 22(1), 287-291.

Moccia, M., Lanzillo, R., Palladino, R., Maniscalco, G.T., De Rosa, A., Russo, C., Massarelli, M., Carotenuto, A., Postiglione, E., Caporale, O., Triassi, M., Brescia Morra, V., 2015. The Framingham cardiovascular risk score in multiple sclerosis. Eur J Neurol 22(8), 1176-1183.

Mosca, L., Barrett-Connor, E., Wenger, N.K., 2011. Sex/gender differences in cardiovascular disease prevention: what a difference a decade makes. Circulation 124(19), 2145-2154.

Motl, R.W., Snook, E.M., Wynn, D.R., Vollmer, T., 2008. Physical activity correlates with neurological impairment and disability in multiple sclerosis. J Nerv Ment Dis 196(6), 492-495.

Pilutti, L.A., Motl, R.W., 2016. Body Mass Index Underestimates Adiposity in Persons With Multiple Sclerosis. Arch Phys Med Rehabil 97(3), 405-412.

Sarafidis, P.A., Lasaridis, A.N., Nilsson, P.M., Pikilidou, M.I., Stafilas, P.C., Kanaki, A., Kazakos, K., Yovos, J., Bakris, G.L., 2007. Validity and reproducibility of HOMA-IR, 1/HOMA-IR, QUICKI and McAuley's indices in patients with hypertension and type II diabetes. Journal of human hypertension 21(9), 709-716. 
Savci, S., Inal-Ince, D., Arikan, H., Guclu-Gunduz, A., Cetisli-Korkmaz, N., Armutlu, K., Karabudak, R., 2005. Six-minute walk distance as a measure of functional exercise capacity in multiple sclerosis. Disabil Rehabil 27(22), 1365-1371.

Warren, T.Y., Barry, V., Hooker, S.P., Sui, X., Church, T.S., Blair, S.N., 2010. Sedentary behaviors increase risk of cardiovascular disease mortality in men. Med Sci Sports Exerc 42(5), 879-885.

Washburn, R.A., Zhu, W., McAuley, E., Frogley, M., Figoni, S.F., 2002. The physical activity scale for individuals with physical disabilities: development and evaluation. Arch Phys Med Rehabil 83(2), 193200.

Wens, I., Dalgas, U., Deckx, N., Cools, N., Eijnde, B., 2013a. Does multiple sclerosis affect glucose tolerance? Mult Scler 20(9), 1273-1276.

Wens, I., Dalgas, U., Stenager, E., Eijnde, B.O., 2013b. Risk factors related to cardiovascular diseases and the metabolic syndrome in multiple sclerosis - a systematic review. Mult Scler 19(12), 15561564.

Wens, I., Dalgas, U., Vandenabeele, F., Krekels, M., Grevendonk, L., Eijnde, B.O., 2014. Multiple sclerosis affects skeletal muscle characteristics. PLoS One 9(9), e108158. 